\title{
Protective Effect of Green Algae Ulva reticulata Against Pseudomonas aeruginosa in Drosophila Infection Model
}

\author{
Firzan Nainu*, Rangga Meidianto Asri, M. Natsir Djide, Muhammad Ahsan, Rudi Arfiansyah, Sartini Sartini, Gemini \\ Alam
}

Faculty of Pharmacy, Hasanuddin University, Makassar, Indonesia

ARTICLE INFO

Article history:

Received March 5, 2019

Received in revised form September 6, 2019

Accepted September 30, 2019

KEYWORDS:

Green algae,

Antipseudomonal,

Fruit flies,

Diptericin,

Drug discovery

\begin{abstract}
The emergence of antibiotic-resistant Pseudomonas aeruginosa is one of main health issues in global communities. To overcome such threat, the discovery of novel antibacterial agents is indispensable. This study aimed to evaluate the in vivo antipseudomonal activity of Ulva reticulata extract in Drosophila model of infection. Ethanolic extract of Ulva reticulata was prepared using maceration method and the extract was subsequently assessed for its in vivo antibacterial effect against $P$. aeruginos $a$ using survival assay, bacterial load enumeration, and gene expression analysis in the wildtype Drosophila. Survival and bacterial load analysis were further performed in a similar fashion on the mutant flies devoid of component responsible in the activation of immune responses against $P$. aeruginosa. Decline in the survival of infected host accompanied by augmentation of bacterial proliferation was documented in the wildtype Drosophila upon infection with $P$. aeruginosa. These phenotypic events were further amplified in immune-deficient mutant Drosophila. Nevertheless, improvement of host survivorship and reduction of bacterial burden were demonstrated in both wildtype or immune-deficient mutant flies upon treatment with Ulva reticulata extract after bacterial challenge. Our data demonstrated in vivo antipseudomonal activity of Ulva reticulata extract and thus provide a valuable information about its future potential for health promotion.
\end{abstract}

\section{Introduction}

The emergence of pathogenic bacteria that are resistant to antibiotics presents a serious problem to the stability of public health (Levy and Marshall 2004; Gelband et al. 2015). One of such bacteria is Pseudomonas aeruginosa. Although P. aeruginosa lives commensally in the skin and mucous membranes, this pathogen most often causes primary infection through air contact in open wounds and/or nosocomial infections through the installation of medical devices (Mesaros et al. 2007). Currently, the use of appropriate antibiotics is the main choice to overcome cases of infection due to antibioticresistant $P$. aeruginosa. However, with the limited selection of functional antibiotics (Luepke et al. 2017; WHO 2017), the discovery of novel antibacterial agents that are meticulously effective against $P$. aeruginosa is urgently required.

\footnotetext{
* Corresponding Author

E-mail Address: firzannainu@unhas.ac.id
}

To date, antibacterial drug discovery was achieved through the exploration of numerous potential sources (Lewis 2013; Wright 2014). One of promising sources for such efforts is green algae Ulva reticulata. This marine green alga belongs to the cosmopolitan genus Ulva and it is widely distributed in the tropical parts of Indowest-Pacific region, including Indonesia (Tilmann et al. 2004; Tsai et al. 2004; Takeshi et al. 2005; Yabe et al. 2009). Whilst the unsolicited presence of Ulva spp. in the coastal regions has been reported to have a detrimental effect on the social and tourism activities (Leliaert et al. 2009; Yabe et al. 2009), different preparations of Ulva reticulata has been traditionally used as nutritional sources (Ratana-arporn and Chirapart 2006; Kim et al. 2011) and nowadays has been suggested to have numerous health benefits (Balaji et al. 2004; Hong et al. 2007; Hong et al. 2011). In addition, recent reports described that ethanolic extract of Ulva reticulata demonstrated excellent in vitro activities against several human pathogenic bacteria, including $P$. aeruginosa (AlSaif et al. 2014; Ravikumar et al. 2016). Unfortunately, 
despite such potential, little information is available regarding the in vivo antibacterial activity of this particular marine species against $P$. aeruginosa.

We recently reported the use of a rapid and lowcost in vivo fruit fly (Drosophila melanogaster) infection platform to examine the antibacterial potential of green algae Ulva reticulata (Nainu et al. 2018) and roselle (Hibiscus sabdariffa L.) calyx (Ahsan et al. 2019) extracts against Staphylococcus aureus. With the use of two straightforward assays: determination of fly survivorship and enumeration of bacterial colonies recovered from fly samples, we compared the susceptibility of the wild type and immune-deficient fruit flies to $S$. aureus infection in the presence or absence of treatment. Indeed, D. melanogaster has been acclaimed as one of appropriate model organisms in the discovery of evolutionally conserved immune-related effectors and signaling pathways in the infection caused by bacteria (Hoffman and Reichhart 2002; Buchon et al. 2014; Panayidou et al. 2014) and viruses (Mussabekova et al. 2017; Nainu et al. 2017; Swevers et al. 2018).

To date, fruit fly has been used at the forefront of modern medical sciences to study biological events that are critically important in the human diseases (Pandey and Nichols 2011; Ugur et al. 2016). With a relatively high degree of genetic similarity with human (Pandey and Nichols 2011) and the fact that D. melanogaster was shown as an appropriate host for P. aeruginosa (D'Argenio et al. 2001; Apidianakis and Rahme 2009; Mulcahy et al. 2011; Haller et al. 2014), this particular insect offers huge and relevant benefits as an in vivo model organism in the field of antimicrobial drug discovery (Chamilos et al. 2011; Tzelepis et al. 2013; Nainu 2018). What is more, the application of D. melanogaster as a model organism in the in vivo experiments offers a great deal of simplicity to work with large number of animals at the same time, requires small attention in the stock keeping, and raises little or even no issues in the ethical point of view (Pandey and Nichols 2011; Ugur et al. 2016). With all of these advantages, we carried out an experimental research to investigate the in vivo antipseudomonal activity of green algae Ulva reticulata.

\section{Materials and Methods}

\subsection{Bacterial Strains and Fly Stocks}

Culture of $P$. aeruginosa ATCC 27853 strain was used as infectious agent in all bacterial infection experiments performed in this study. Appropriate inoculum of bacteria was prepared by culturing the bacteria in Nutrient Broth (NB) medium at $37^{\circ} \mathrm{C}$ for $1 \times 24$ hours and processed thoroughly by a series of washing with PBS prior to quantification using spectrophotometry method. Bacterial inoculum was freshly prepared in PBS for each experiment. The following fly lines were used in this study: $w^{1118}$ as genetic background (control) line and imd $^{1}$ as immune-deficient fly line with undetected level of Imd (both Drosophila lines were generously provided by Prof. Yoshinobu Nakanishi, Kanazawa University). Male flies were used in the entire experiments and they were anesthetized with $\mathrm{CO}_{2}$ prior to carrying out bacterial infection experiments. Flies were maintained in standard conditions $\left(25^{\circ} \mathrm{C}, 12: 12 \mathrm{~h}\right.$ light:dark cycle, standard cornmeal-agar food).

\subsection{Plant Extract Preparation}

Fresh samples of the green algae Ulva reticulata were obtained from Puntondo, Takalar, South Sulawesi, Indonesia and processed immediately as previously described by Al-Saif et al. (2014), with slight modifications. Green algae samples were sorted and subjected to maceration procedures using $96 \%$ ethanol for $3 \times 24$ hours and re-macerated for $1 \times 24$ hours. The resulting filtrates were collected and further processed using rotavapor to reduce the water content. The process was carried out thoroughly until extract with appropriate thickness were successfully obtained. The resulting extract was subsequently kept in a brown silica container prior to use.

\subsection{Fly Infection and Assays for Survival and Bacterial Growth}

All bacterial infection experiments were carried out using pricking method, as described previously (Nainu et al. 2018; Ahsan et al. 2019). In this method, adult male flies at age of 4-7 days after eclosion (15 flies per vial, 3 vials per group in each experiment) were subjected to infection procedure in which bacteria were introduced into the thorax of adult flies $\left(0.9 \times 10^{5} \mathrm{cfu} / \mathrm{ml}\right.$ per fly) and flies were further maintained in incubator with steady temperature at $29^{\circ} \mathrm{C}$. A group of mockinfected flies were prepared as a control group in each infection experiment. These flies were similarly treated but pricked with PBS instead of bacterial inoculum. Mock-infected and bacterial-infected flies were then subsequently subjected to either survival assay or colony forming assay as described previously (Nainu et al. 2018; Ahsan et al. 2019). In the survival assay, groups of flies were pricked with either P. aeruginosa 
or PBS and maintained in the presence or absence of treatments. Daily observation was carried out to record the number of live flies at each group as designated. In the colony forming assay, groups of flies were subjected to similar procedures as in the survival assay but at a given time, live flies were taken from each group and mechanically processed using a micropestle in PBS solution. Homogenates obtained from all groups were independently prepared at serial dilutions, plated on Cetrimide agar (CETA) medium, and incubated at $37^{\circ} \mathrm{C}$ for 1 x 24 hours. Number of colonies appeared after incubation was expressed as cfu per $\mathrm{ml}$.

\subsection{Gene Expression Analysis}

Isolation of total RNA was carried out on designated groups of treatments. In this assay, five live Drosophila were collected from each group at 50 hours post infection and transferred into Treff tubes. Flies were then homogenized in the Treff tube using a micropestle prior to further processing using SV Total RNA Isolation System (Promega) according to the manufacturer's protocol. Level of diptericin (Dpt) was quantitatively determined in the RNA preparations using reverse transcriptase quantitative PCR (RT-qPCR) method based on the use of one set of $D p t$ primer (sequence of $D p t$ forward primer: 5'-GCTGCGCAATCGCTTCTACT-3' and sequence of Dpt reverse primer: 5'-TGGTGGAGTGGGCTTCATG-3') in a $20 \mu$ reaction volume using GoTaq ${ }^{\circledR}$ 1-Step RT-qPCR System (Promega), as per manufacturer's instruction. Rotor-Gene Q thermal cycler (Qiagen, Germany) was used with the following profile: $37^{\circ} \mathrm{C}$ for $15 \mathrm{mins}, 95^{\circ} \mathrm{C}$ for $10 \mathrm{mins}$, and $40 \mathrm{cycles}$ of $95^{\circ} \mathrm{C}$ for $10 \mathrm{~s}$ and $60^{\circ} \mathrm{C}$ for $30 \mathrm{~s}$, and $72^{\circ} \mathrm{C}$ for $30 \mathrm{~s}$. To confirm that only the expected product had been amplified, a standard melt curve analysis was carried out in every run. Using a similar RTqPCR protocol, RNA level of host ribosomal protein rp49 (used as an internal control) was examined by using one set of rp49 primer (sequence of rp49 forward primer: 5'-GACGCTTCAAGGGACAGTATCTG-3' and sequence of rp49 reverse primer: 5'-AAACGCGGTTCTGCATGA G-3'). The relative abundance of $D p t$ to the host reference gene rp49 was analyzed using qGENE software.

\subsection{Data Processing and Statistical Analysis}

In this study, results obtained from at least three independent biological replicates were statistically analyzed using GraphPad Prism ${ }^{\circledR}$ 7. Survival of flies during infection was displayed as a Kaplan-Meier curve and analysed using log-rank analysis. For results obtained in the CFU and gene expression experiments, data were prepared as bar graphs and analyzed statistically using one-way ANOVA. Data from all experiments are presented as mean \pm S.D and $p$ values of less than 0.05 were considered significant.

\section{Results}

\subsection{Reproduction of Drosophila Model of $\boldsymbol{P}$. aeruginosa Infection}

Apidianakis and Rahme previously published a procedure for the infection of $D$. melanogaster with $P$. aeruginosa. They suggested that flies can be inoculated with $P$. aeruginosa either by feeding, needle pricking, or injection. Based on the ease of technique and availability of equipment, we decided to use the needle pricking technique. In this study, we infected 4-7 days of male D. melanogaster with $P$. aeruginosa, a Gram-negative bacterium that have been reported to cause multitude of clinically relevant diseases on humans and other species such as nematodes and insects, including $D$. melanogaster. We selected one characteristic, alterations in the flies' survival rate, as an indication of the infection success. As shown in Figure 1, infection of D. melanogaster $w^{1118}$ with different concentration of $P$. aeruginosa stocks resulted in the dose-dependent reduction of flies' survival rate, suggesting that the infection condition was successfully established in all three independent experiments and the needle pricking

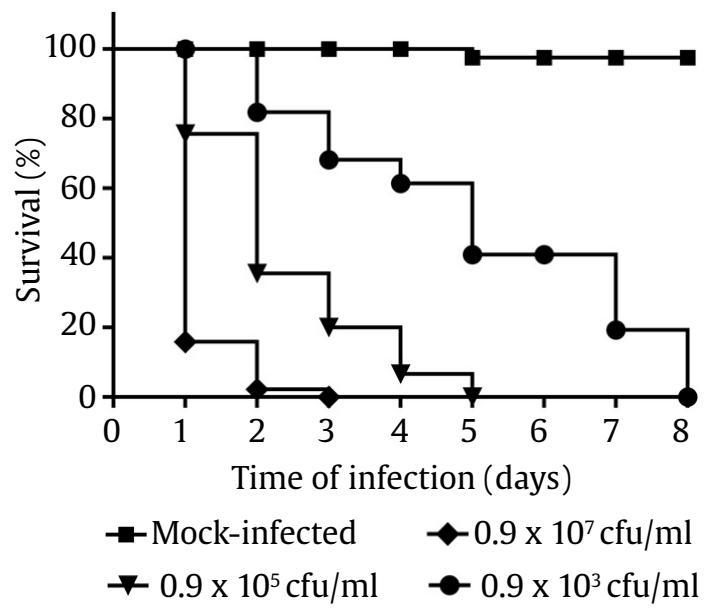

Figure 1. Dose-response of $P$. aeruginosa in the infection experiment. (a) Adult $w^{1118}$ flies at 4-7 days after eclosion were infected with a range of doses of $P$. aeruginosa by pricking and subjected to fly survival analysis. (b) Adult $w^{1118}$ flies at 4-7 days after eclosion were infected with $0.9 \mathrm{x}$ $10^{5} \mathrm{cfu} / \mathrm{ml}$ of $P$. aeruginosa (PA) by pricking and subjected to fly survival analysis in the presence of tetracycline (TET) at different concentrations 
technique was sufficient to yield reproducible results, at least in our hands.

In this experiment, we intended to use D. melanogaster as an in vivo platform to assess the antibacterial effect of green algae Ulva reticulata on P. aeruginosa. However, we have not established a proper antibiotic to be used as a positive control for our infection experiments. We previously confirmed that treatment of $S$. aureusinfected Drosophila $w^{1118}$ with tetracycline, a potent bacteria protein synthesis inhibitor, was sufficient to prevent host early death phenotype. Since tetracycline is a broad-acting antibiotic, we believed that this drug would also provide antibacterial protection against $P$. aeruginosa in $D$. melanogaster. To assess the effect of tetracycline on the survival of infected flies, we incubated $P$. aeruginosa-infected $D$. melanogaster in a vial with food containing tetracycline. Indeed, tetracycline treatment prevented early death phenotype on the infected flies (data not shown), thus confirmed its suitability to be used as the positive control in our further infection experiments.

\subsection{Dose-dependent Effect of Ulva reticulata Extract in The Survival of Adult Drosophila melanogaster}

Next, we carried out a survival assay to examine the possible antibacterial effect of green algae Ulva reticulata extract against $P$. aeruginosa. In this experiment, we infected adult males of $w^{1118}$ flies with $0.9 \times 10^{5} \mathrm{cfu} /$ $\mathrm{ml}$ of $P$. aeruginosa and incubated them at $29^{\circ} \mathrm{C}$ in the presence or absence of Ulva reticulata extract at different concentrations. As shown in Figure 2a, flies were succumbed to immediate death upon $P$. aeruginosa infection and apparently, the survival of infected flies was not improved if maintained in foods containing Ulva reticulata extract at lower concentrations ( 0.1 and $0.5 \% \mathrm{w} / \mathrm{w}$ ). Nevertheless, the survivorship of infected flies became greatly enhanced once maintained in the presence of Ulva reticulata extract at a concentration as much as $2.5 \% \mathrm{w} / \mathrm{w}$ or tetracycline. From this experiment we demonstrated that the presence of certain concentration of Ulva reticulata extract in the food was sufficient to rescue $P$. aeruginosa-infected $D$. melanogaster from early death phenotype, indicating the in vivo antibacterial effect of Ulva reticulata extract against $P$. aeruginosa was achieved in a dose-dependent manner.

\subsection{Inhibition of Pseudomonas aeruginosa Growth by Green Algae Ulva reticulata}

Increased mortality of $D$. melanogaster upon bacterial infection has been shown to be linearly correlated with increased bacterial load thus inhibition of bacterial growth by antibacterial agent was one of the ways to improve the survival of infected host. In support to this notion, we expected the reduction of pathogenic burden ( $P$. aeruginosa load) will be demonstrated by the infected flies treated with either tetracycline (as a positive control) or $2.5 \%$ Ulva reticulata extract. To assess this, we carried out a simple colony forming assay using Cetrimide agar (CETA) as the specific growth medium for $P$. aeruginosa. As a result of this assay, we found that treatment of $P$. aeruginosa-infected flies with either tetracycline or Ulva reticulata extract was greatly useful to reduce the bacterial load (Figure 2b), suggesting that bacterial growth inhibition by tetracycline or Ulva reticulata extract could lead to the increased survivorship of infected flies.

\subsection{Ulva reticulata-mediated Antibacterial Protection is Independent of Diptericin Overstimulation}

In response to infection by Gram-negative bacteria, Drosophila expressed a number of genetically-encoded antimicrobial peptides (AMP) that functionally work as a part of its innate immune responses. Taken this into account, we speculated that protection of infected flies observed in the group treated with Ulva reticulata extract might be achieved either by direct antibacterial effect against $P$. aeruginosa or by overstimulation of specific host immune responses. Of the known AMPs in Drosophila, a particular AMP named as Diptericin, which is encoded by the diptericin (dpt) gene, has been suggested to play a significant role against Gram-negative bacteria such as $P$. aeruginosa. To confirm whether overexpression of Diptericin plays a part in the host protection against $P$. aeruginosa upon treatment with Ulva reticulata extract, we assessed the expression level of diptericin in all designated groups. As shown in Figure 3, we detected an increased expression of diptericin in samples prepared from flies infected with $P$. aeruginosa, clearly indicating that Diptericin is expressed in a manner dependent on infection. Moreover, treatment of $P$. aeruginosainfected flies with either tetracycline or Ulva reticulata extract resulted in the reduced expression of diptericin, further clarifying the insignificant role of Diptericin in Ulva reticulata-mediated protection of Drosophila against $P$. aeruginosa.

\subsection{Protection of Immune-deficient Drosophila Against $P$. aeruginosa by Ulva reticulata Extract}

We observed that green algae Ulva reticulata extract exerts its antibacterial effect in the wildtype host upon infection with $P$. aeruginosa and such protection was not due to the overstimulation of 

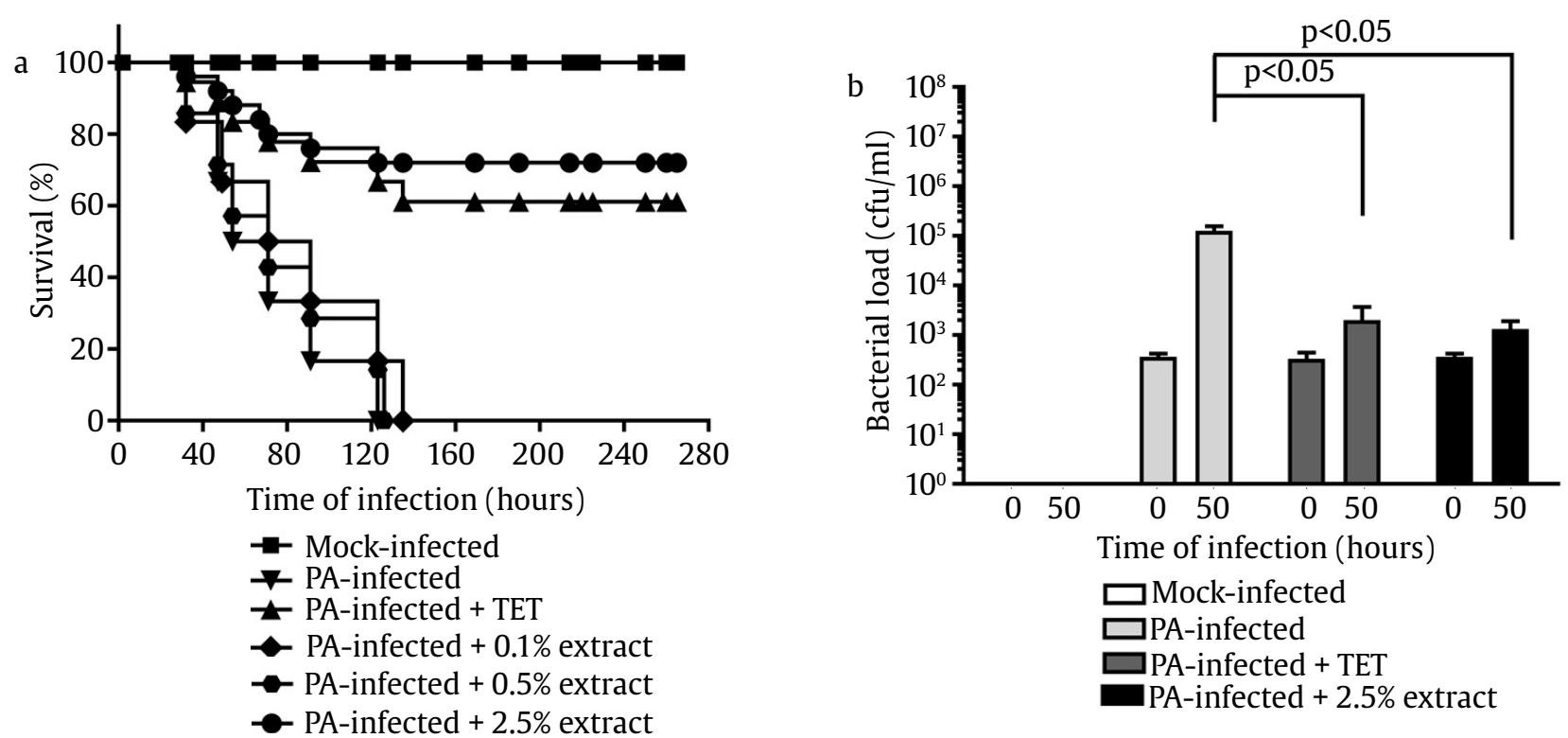

Figure 2. Improvement of host survival and reduced bacterial load in infected flies in the presence Ulva reticulata extract. Adult $w^{1118}$ flies at 4-7 days after eclosion were infected with $0.9 \times 10^{5} \mathrm{cfu} / \mathrm{ml}$ of $P$. aeruginosa (PA) by pricking, incubated at $29^{\circ} \mathrm{C}$ in the presence of $2.5 \%$ Ulva reticulata extract, and subjected to fly survival (a) and bacterial load (b) analysis. Flies treated with tetracycline (TET) at $200 \mu \mathrm{g} / \mathrm{ml}$ were used as a positive control group

Diptericin, a specialized AMP commonly expressed in D. melanogaster to fight against Gram-negative bacteria, including $P$. aeruginosa. Based on these results, we believed that Ulva-reticulata-mediated antibacterial protection was achieved from direct interaction between antibacterial compounds contained in the extract and $P$. aeruginosa components in infected flies. To further support this view with data, we carried out infection experiments on immune-deficient flies lacking for Imd pathway that has been previously shown to have impaired production of Diptericin. As shown in Figure 4, we observed that the survival rate of $i m d^{1}$ mutant flies was dramatically declined upon $P$. aeruginosa infection (Figure 4a) and these immunodeficient flies tend to contain high bacterial load, around 100 -folds higher than their mock-infected counterparts (Figure 4b). Nonetheless, treatment of $P$. aeruginosa-infected immune-deficient fly line lacking for Imd pathway with either tetracycline or Ulva reticulata extract at concentration of $2.5 \%(\mathrm{w} / \mathrm{w})$ was deliberately adequate to improve flies' survivorship (Figure 4a) and significantly helpful to reduce bacterial growth (Figure 4b) during the time of infection. These results further clarified that antipseudomonal activity of Ulva reticulata extract in adult Drosophila was not due to the involvement of Imd-mediated production of Diptericin.

\section{Discussion}

Pseudomonas aeruginosa has been famously known as one of pathogenic bacteria with considerable medical importance (Mesaros et al. 2007). In fact, accumulating evidence has suggested that some of the $P$. aeruginosa isolates develop the ability to resist the effect of a broad range of antibiotics, termed as antibiotic-resistant $P$. aeruginosa (Mesaros et al. 2007; Jorgensen et al. 2015), thus jeopardizing our efforts to maintain the public health stability in the world. With such threat, $P$. aeruginosa would be an appropriate target for antibacterial drug discovery

Pseudomonas aeruginosa is a bacilliform, facultative anaerobe, Gram-negative bacterium that is easily characterized by the presence of two specific metabolites-pyocyanin and pyoverdine-that gives the detectable blue-green color characteristics on the Cetrimide agar culture plate (Jorgensen et al. 2015). The production of bright green color due to combinatory reaction between fluorescent pigment pyoverdin and blue hydrophilic pigment pyocyanin has been used as a positive indication of the $P$. aeruginosa presence in the tested samples (Jorgensen et al. 2015).

Recently, we demonstrated the use of adult $D$. melanogaster as a novel, straightforward and low-cost in vivo platform to examine the antibacterial activity of Ulva reticulata extract (Nainu et al. 2018) and roselle 

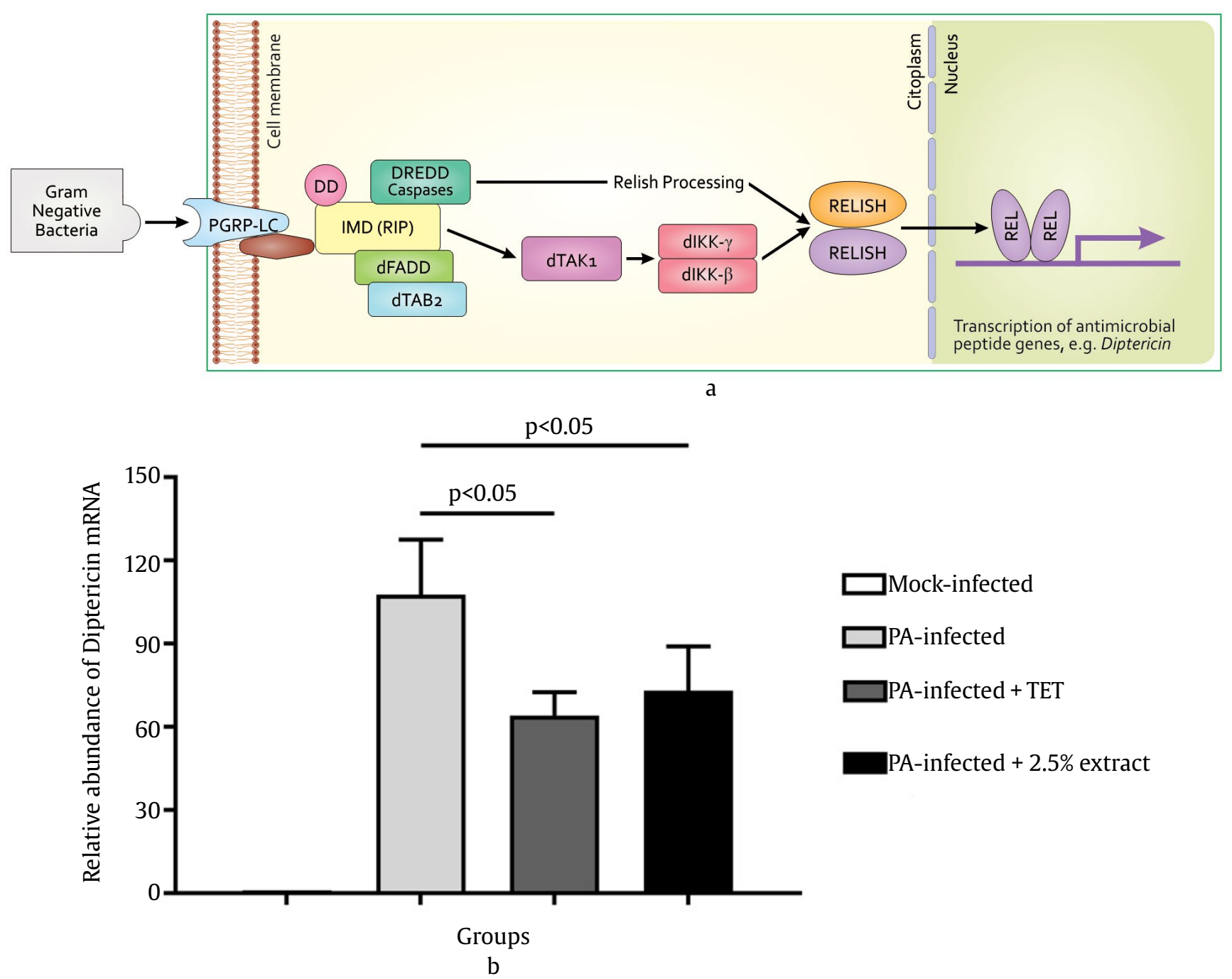

Figure 3. Anti-pseudomonal protection of Ulva reticulata extract was achieved independent of Diptericin stimulation. (a) upon infection with Gram-negative bacteria, Diptericin is expressed in response to activation of Imd pathways in Drosophila. (b) adult $w^{1118}$ flies at 4-7 days after eclosion were infected with $0.9 \times 10^{5} \mathrm{cfu} / \mathrm{ml}$ of $P$. aeruginosa (PA) by pricking, incubated at $29^{\circ} \mathrm{C}$ in the presence of $2.5 \%$ Ulva reticulata extract, and subjected to RNA isolation followed by Diptericin mRNA level quantification by RT-qPCR. Expression of reference gene rp 49 was used as the internal control. Flies treated with tetracycline (TET) at $200 \mu \mathrm{g} / \mathrm{ml}$ were used as a positive control group

(Hibiscus sabdariffa L.) calyx extract (Ahsan et al. 2019) against $S$. aureus infection. Here, we employed such in vivo platform to assess the antipseudomonal activity of Ulva reticulata extract. We used similar approaches: assessment of fly survival assays and numerical determination of colony forming bacteria, to investigate the antibacterial effect of Ulva reticulata extract against $P$. aeruginosa infection. In conjunction with molecular analysis to examine the expression of Diptericin, a well-known Drosophila immune response against Gram-negative bacteria, data obtained in this study clearly indicated the antipseudomonal activity of Ulva reticulata extract and such antibacterial protection was achieved independent of Diptericin-mediated immune responses in the affected host. In addition to that, direct comparison of survival rate and bacterial load analysis between $w^{1118}$ and $i m d^{1}$ flies further validates the important role of Imd-mediated protection against $P$. aeruginosa infection, as reported by others (D'Argenio et al. 2001; Limmer et al. 2011). It would be interesting to see in the future research whether different components of the Imd signaling pathway and/or other antibacterial-related pathways such as Toll and JAKSTAT pathways are overstimulated by the infected host in response to the ingestion of Ulva reticulata extract. Furthermore, while our data suggested the protective 


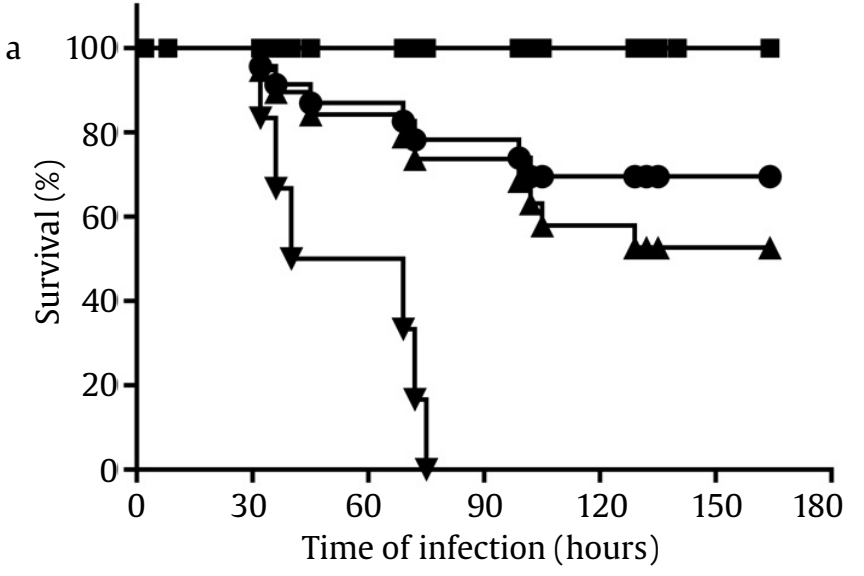

$$
\begin{aligned}
& \rightarrow \text { - Mock-infected } \rightarrow \text { PA-infected } \\
& \neg \text { PA-infected + TET } \longrightarrow \text { - PA-infected }+2.5 \% \text { extract }
\end{aligned}
$$

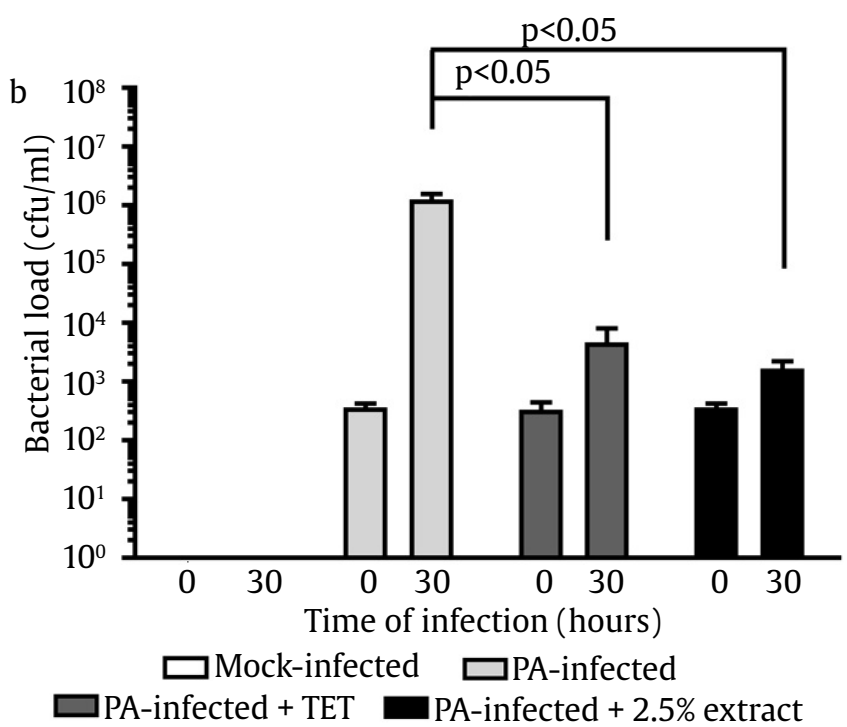

Figure 4. Enhancement of host survivorship and impaired bacterial proliferation in immunodeficient flies in the presence of Ulva reticulata extract. Adult imd ${ }^{1}$ flies at 4-7 days after eclosion were infected with $0.9 \times 10^{5} \mathrm{cfu} / \mathrm{ml}$ of $P$. aeruginosa (PA) by pricking, incubated at $29^{\circ} \mathrm{C}$ in the presence of $2.5 \%$ Ulva reticulata extract, and subjected to fly survival (a) and bacterial load (b) analysis. Flies treated with tetracycline (TET) at $200 \mu \mathrm{g} / \mathrm{ml}$ were used as a positive control group

effect of Ulva reticulata extract against $P$. aeruginosa in $D$. melanogaster, compound(s) responsible for such antibacterial effect are unknown. Certainly, the elucidation of compounds with antibacterial properties in Ulva reticulata extract is one of important directions to pursue in the future studies.

The combination of two phenotypic analysis used in this study, survivorship of infected flies and enumeration of bacteria recovered from infected flies, has been recognized as a simple yet efficient way to investigate the antimicrobial properties of potential samples (Apidianakis and Rahme 2009; Nainu et al. 2018; Ahsan et al. 2019). By using this approach, researchers will be able to obtain appropriate compounds with in vivo activities at the initial screening and at the same time will be able to exclude compounds that are highly toxic to the eukaryotic host cells. In addition to that, a high degree of genetic similarity between $D$. melanogasterand human (around 75\%) suggests a reasonable likelihood of getting similar results in the clinical trials of the respective drug candidates. Certainly, all advantages offered by Drosophila in vivo infection platform used in this study shall be a great rationale for researchers before moving to the next stage using mammalian animal models. Such approach will eventually support the rapid advancement of antimicrobial drug discovery in an inexpensive and logical manner.

\section{Conclusion}

In this study, for the first time, we confirmed the in vivo antipseudomonal activity of green algae Ulva reticulata extract using a novel, straight-forward and low-cost Drosophila platform system. This model is based on two phenotypic results: survival rate improvement and reduction of bacterial load, followed by the use of molecular analysis to rule out the potential immunostimulation activity of the assessed sample. With this data in hand, we further demonstrated the relevance of $D$. melanogaster as an in vivo platform to investigate the potential antibacterial activity of a particular sample of interest. With the abundance resources in Indonesia and other countries, such platform would serve as a great inexpensive in vivo approach to rapidly discover important antibacterial compounds from multiple sources of interests in no time.

\section{Acknowledgements}

This research was financially supported by Penelitian Percepatan Publikasi Internasional World Class University (PPI-WCU) research grant from Hasanuddin University (to F.N, RMA, and MND). We would like to offer our gratitude to Prof. Yoshinobu Nakanishi of Kanazawa 
University for the provision of Drosophila lines and Dr. Isra Wahid of Hasanuddin University for his kind help in the measurement of total RNA concentration in adult fly samples. The use of FlyBase is fully acknowledged.

\section{References}

Ahsan $\mathrm{M}$ et al. 2019. In vivo anti-staphylococcal activity of roselle (Hibiscus sabdariffa L.) calyx extract in Drosophila model of infection.J Herbmed Pharmacol 8:41-46.

Al-Saif SSA-l et al. 2014. Antibacterial substances from marine algae isolated from Jeddah coast of Red sea, Saudi Arabia. Saudi J Biol Sci 21:57-64.

Apidianakis Y, Rahme LG. 2009. Drosophila melanogaster as a model host for studying Pseudomonas aeruginosa infection. Nat Protoc 4:1285-1294.

Balaji RRH et al. 2004. Antihepatotoxic nature of Ulva reticulata (Chlorophyceae) on acetaminophen-induced hepatoxicity in experimental rats.J Med Food 7:495-497.

Buchon Net al. 2014. Immunity in Drosophila melanogaster-from microbial recognition to whole-organism physiology. Nat Rev Immunol 14:796-810.

Brown VI, Lowbury EJL. 1965. Use of an improved cetrimide agar medium and other culture methods for Pseudomonas aeruginosa. J Clin Pathol 18:752-756.

Chamilos G et al. 2011. Drosophila melanogaster as a model host for the study of microbial pathogenicity and the discovery of novel antimicrobial compounds. Curr Pharm Des 17:1246-1253.

D'Argenio DA et al. 2001. Drosophila as a model host for Pseudomonas aeruginosa Infection.J Bacteriol 183:14661471.

Gelband $\mathrm{H}$ et al. 2015. The State of the World's Antibiotics, 2015. Washington D.C: The Center for Disease Dynamics, Economics and Policy.

Haller S et al. 2014. Assessing Pseudomonas virulence with a nonmammalian host: Drosophila melanogaster. In: Filloux A, Ramos J-L (Eds.). Pseudomonas Methods and Protocols. New York: Springer. pp. 723-40.

Hoffmann JA, Reichhart JM. 2002. Drosophila innate immunity: an evolutionary perspective. Nat Immunol 3:121-126.

Hong DD et al. 2007. Seaweeds from Vietnam used for functional food, medicine and biofertilizer. J Appl Phycol 19: 817-826.

Hong DD et al. 2011. Studies on the analgesic and antiinflammatory activities of Sargassum swartzii (Turner) C. Agardh (Phaeophyta) and Ulva reticulata Forsskal (Chlorophyta) in experiment animal models. Afr J Biotechnol 10:2308-2314.

Jorgensen JH et al. 2015. Manual of Clinical Microbiology. $11^{\text {th }}$ ed. New York:ASM Press.

Kim SK et al. 2011. Sea lettuces: culinary uses and nutritional value. In: Kim SK (Eds). Advances in Food and Nutrition Research. Vol. 64. New York: Academic Press. pp. 57-70.

Leliaert F et al. 2009. Identity of the Qingdao algal bloom. Phycol Res 57:147-151.

Lemaitre B, Hoffmann J. 2007. The host defense of Drosophila melanogaster. Annu Rev Immunol 25:697-743.
Levy SB, Marshall B. 2004. Antibacterial resistance worldwide: causes, challenges and responses. Nat Med 10:122-129.

Lewis K. 2013. Platforms for antibiotic discovery. Nat Rev Drug Discov 12:371-387.

Limmer Set al. 2011. Pseudomonas aeruginosa RhIR is required to neutralize the cellular immune response in a Drosophila melanogaster oral infection model. Proc Natl Acad Sci 108:17378-17383.

Luepke KH et al. 2017. Past, present, and future of antibacterial economics: increasing bacterial resistance, limited antibiotic pipeline, and societal implications. Pharmacother J Hum Pharmacol Drug Ther 37:71-84.

Mesaros N et al. 2007. Pseudomonas aeruginosa: resistance and therapeutic options at the turn of the new millennium. Clin Microbiol Infect 13:560-578.

Mulcahy H et al. 2011. Drosophila melanogaster as an animal model for the study of Pseudomonas aeruginosa biofilm infections in vivo. PLoS Pathog 7:e1002299.

Mussabekova A et al. 2017. Innate and intrinsic antiviral immunity in Drosophila. Cell Mol Life Sci 74:2039-2054.

Nainu F et al. 2017. Induction of apoptosis and subsequent phagocytosis of virus-infected cells as an antiviral mechanism. Front Immunol 8:1-11.

Nainu $\mathrm{F}$ et al. 2018. In vivo antibacterial activity of green algae Ulva reticulata against Staphylococcus aureus in Drosophila model of infection. PharmacogJ 10:993-997.

Nainu F. 2018. Penggunaan Drosophila melanogaster sebagai organisme model dalam penemuan obat. Galenika J Pharm 4:50-67.

Needham AJ et al. 2004. Drosophila melanogaster as a model host for Staphylococcus aureus infection. Microbiol 150:2347-2355.

Pandey UB, Nichols CD. 2011. Human disease models in Drosophila melanogaster and the role of the fly in therapeutic drug discovery. Pharmacol Rev 63: 411-436.

Panayidou S et al. 2014. Human pathogenic bacteria, fungi, and viruses in Drosophila: disease modeling, lessons, and shortcomings. Virulence 5:253-269.

Ratana-arporn P, Chirapart A. 2006. Nutritional evaluation of tropical green seaweeds Caulerpa lentillifera and Ulva reticulata. Kasetsart J (Nat Sci) 40:75-83.

Ravikumar S et al. 2016. Antibacterial activity of Ulva reticulata from southwest coast of Kanyakumari, India. J Coast Life Med 4:246-247.

Swevers L et al. 2018. Defense mechanisms against viral infection in Drosophila: RNAi and non-RNAi. Viruses 10:230-265.

Takeshi S et al. 2005. Mineral components and anti-oxidant activities of tropical seaweeds. J Ocean Uni China 4:205-208.

Tilmann H et al. 2004. Waterborne polar macromolecules act as algal antifoulants in the seaweed Ulva reticulata. Mar Ecol Prog Ser 274:133-141.

Tsai C-C et al. 2004. Macroalgal assemblage structure on a coral reef in Nanwan Bay in southern Taiwan. Bot Mar 47:439-453.

Tzelepis I et al. 2013. Drosophila melanogaster: a first step and a stepping-stone to anti-infectives. Curr Opin Pharmacol 13:763-768. 
Ugur B et al. 2016. Drosophila tools and assays for the study of human diseases. Dis Mod Mech 9:235-244.

[WHO] World Health Organization 2017. Global Antimicrobial Resistance Surveillance System(GLASS) Report. Available at:https://www.who.int/glass/resources/publications/ early-implementation-report/en/ [Date accessed: 23 August 2018]
Wright GD 2014. Something old, something new: revisiting natural products in antibiotic drug discovery. Can J Microbiol 60:147-154.

Yabe T et al. 2009. Green tide formed by free-floating Ulva spp. at Yatsu tidal flat, Japan. Limnology 10:239-245. 